\title{
Vertebral level and measurements of conus medullaris and dural sac termination with special reference to the apex of the sacral hiatus: anatomical and magnetic resonance imaging radiologic study
}

\author{
A.Y. Nasr 1,2 \\ ${ }^{1}$ Department of Anatomy, Faculty of Medicine, Zagazig University, Zagazig, Egypt \\ ${ }^{2}$ Department of Anatomy, Faculty of Medicine, King Abdulaziz University, Jeddah, Kingdom of Saudi Arabia
}

[Received: 18 November 2016; Accepted: 30 November 2015]

Background: Anatomical orientation of the caudal space and termination level of conus medullaris (CMT) and dural sac (DST) has great significance for anaesthetists and neurosurgeons. This study aimed to explore the anatomical landmarks important to perform save spinal anaesthesia, lumber puncture and caudal analgesia through the correlation between the vertebral level of CMT, DST and sacral hiatus apex (SHA) in human cadavers and by using magnetic resonance imaging (MRI). Materials and methods: Sixty adult cadavers (40 males, 20 females) and 200 (100 males, 100 females) MR lumbosacral images of 16-69-year-old persons were used in this study. Vertebral level of CMT, DST and SHA and their linear distances were determined in cadavers and MRI. Also, anteroposterior diameter at SHA, length and thickness of sacrococcygeal membrane were measured as well, and correlate these levels and their distances with age and sex.

Results: Mean and highest frequent number of MRI vertebral level of CMT was observed at lower third of L1 in men and L1-2 disc in women, that of DST at upper third of $S 2$ in men and middle third of $S 2$ in women, while SHA was seen at middle third of $S 4$ in both men and women with no significant $(p>0.05)$ age or gender differences. In $5 \%$ of cases, CMT, DST and SHA were seen at vertebral level below L2, below S2-3 and above S3, respectively. However, mean vertebral level of CMT, DST, SHA was observed at L1L, S2M and S4U without sex difference in cadaveric specimens, respectively. All linear distances of men MRI revealed significant difference $(p<0.05)$ compared with those of women except thickness of sacrococcygeal membrane and anterposterior diameter at SHA.

Conclusions: Accurate knowledge of vertebral level of CMT, DST and SHA and the distances in-between might decrease the iatrogenic injury of dural sac, spinal cord and cauda equina. (Folia Morphol 2016; 75, 3: 287-299)

Key words: conus meduularis, dural sac, termination, anatomy, magnetic resonance imaging, level

Address for correspondence: A.Y. Nasr, MD, Anatomy Department, Faculty of Medicine, Zagazig University, Zagazig, Egypt, tel: 002-011151822051; 002-01115277214, 002-055-3763530, fax: 002-055-2307830, e-mail: ashrafnaeem2013@gmail.com; ashrafnaeem2013@yahoo.com

This work is done in: Anatomy Department, Faculty of Medicine, King Abdulaziz University, Jeddah, Kingdom of Saudi Arabia. 


\section{INTRODUCTION}

The conus medullaris (CM) is the caudal tapering end of the spinal cord [26]. Its termination level is considered as an area of interest as it has a great clinical significance in diagnosis of tethered cord syndrome, spinal aneasthesia, myelography and lumber puncture [4]. Magnetic resonance imaging (MRI) provides an accurate information on the different body structures. Thus, the measurements and observations of the position of $\mathrm{CM}$ has become more accurate, accessible and reliable by using MRI [12]. Termination level of $\mathrm{CM}$ is usually observed at lower third of first lumber vertebra (L1L) in most cases, however a wide range of values have been reported in cadaver studies as well as MRI studies $[12,19]$. Few reports has been focused on the effect of age and sex on the termination level of CM [19, 25].

The dural space and sacral hiatus are two regions of great clinical significance. The exploration of these areas needs a good knowledge of their relevant anatomy [26]. The orientation of the relevant anatomy and contents of such spaces is so important especially for spine surgeons and anaesthtests to avoid any accidental injury of their contents [19]. The termination level of dural sac has been observed at second sacral vertebra (S2) $[1,26]$, while a caudal extension beyond this level is reported in other cadaveric studies $[2,9,12,25]$.

The sacral hiatus (SH) is the developmental cuadal defect on the lower most caudal part of sacral canal. It is formed as a result of failure of fusion the laminae of fourth and sometimes fifth sacral vertebrae. This defect is covered by sacrococcygeal membrane (SCM), subcutaneous fatty tissue and skin. SH contains lower sacral and coccygeal nerve roots and fium terminale [26]. SH is an effective site for injection of epidural aneasthesia or analgesia in different clinical specialities namely anaesthesiologists, obstetricians, orthopaedic surgeons, radiologists, neurologists and spine surgeons [27]. The anatomical variations of such structures may contribute to inability to do many diagnostic and therapeutic procedures safely [1]. Thus, knowledge of the vertebral level of sacral hiatus apex (SHA), caudal termination of conus medullaris (CMT) and dural sac termination (DST) is important for successful spinal anaesthesia, lumber puncture and caudal epidural block (CEB) [13].

The aim of this study is to determine the vertebral levels and distances at which the CM terminates, dural sac ends and the SHA in adult human cadaveric specimens and sagittal MR images. Length and thickness SCM and anteroposterior (AP) diameter of sacral canal at the level of SHA were assessed as well. Correlation of these levels and measurements with age and sex was done.

\section{MATERIALS AND METHODS}

Sixty adult human cadaveric specimens (40 males, 20 females) and 200 (100 males, 100 females) MR lumbosacral T1 and T2-weighted sagittal images of 16-69-year-old persons were used in this study. The cadavers were obtained from the Anatomy Department, Faculty of Medicine, King Abdulaziz University, Jeddah, Saudi Arabia. The cadavers were placed in the prone position. A longitudinal midline skin incision was done. The paravertebral muscles were detached to expose the spinous processes and laminae of the vertebrae from T10 to S4. Laminectomy of T11-S4 vertebrae was performed to open the vertebral canal and expose the dural sac. A longitudinal mid-dorsal incision of the dural sac was done throughout the exposed part. The dural sac was also traced down to its termination. The vertebral levels of CMT, DST and SHA were determined. The linear distance between the vertebral level of CMT, DST and SHA as well as the length and thickness of SCM were measured by using a digital Vernier calliper (0.01 mm accuracy) (Fig. 1).

Two hundred MR lumbosacral images of T1- and T2-weighted sagittal spin-echo were done on adult patients at the Diagnostic Radiology Department, King Abdulaziz University hospital, Faculty of Medicine, King Abdulaziz University, Jeddah, Kingdom of Saudi Arabia. The patients were chosen randomly from the patients of the neurology clinics who were referred to MR Unit to assess the possible cause of radiculopathy, sciatica and lower back pain. Patients having congenital anomalies, degenerative lesion, malignancies or previous surgery in the lumbosacral area were excluded from this study. The male and female MRI patients were classified into six age groups, with 10 years apart. The age of first group was $<20$ years (10 patients). Age of the second group was 20-29 years (20 patients); age of third group was 30-39 years (20 patients); age of fourth group was 40-49 years (20 patients), age of fifth group was $50-59$ years (20 patients) and the age of sixth group was $\leq 60$ years (10 patients).

GE Signa 0.5 Tesla MR Machine scanner (GE Medical Systems, Milwaukee, USA) was used to create sag- 

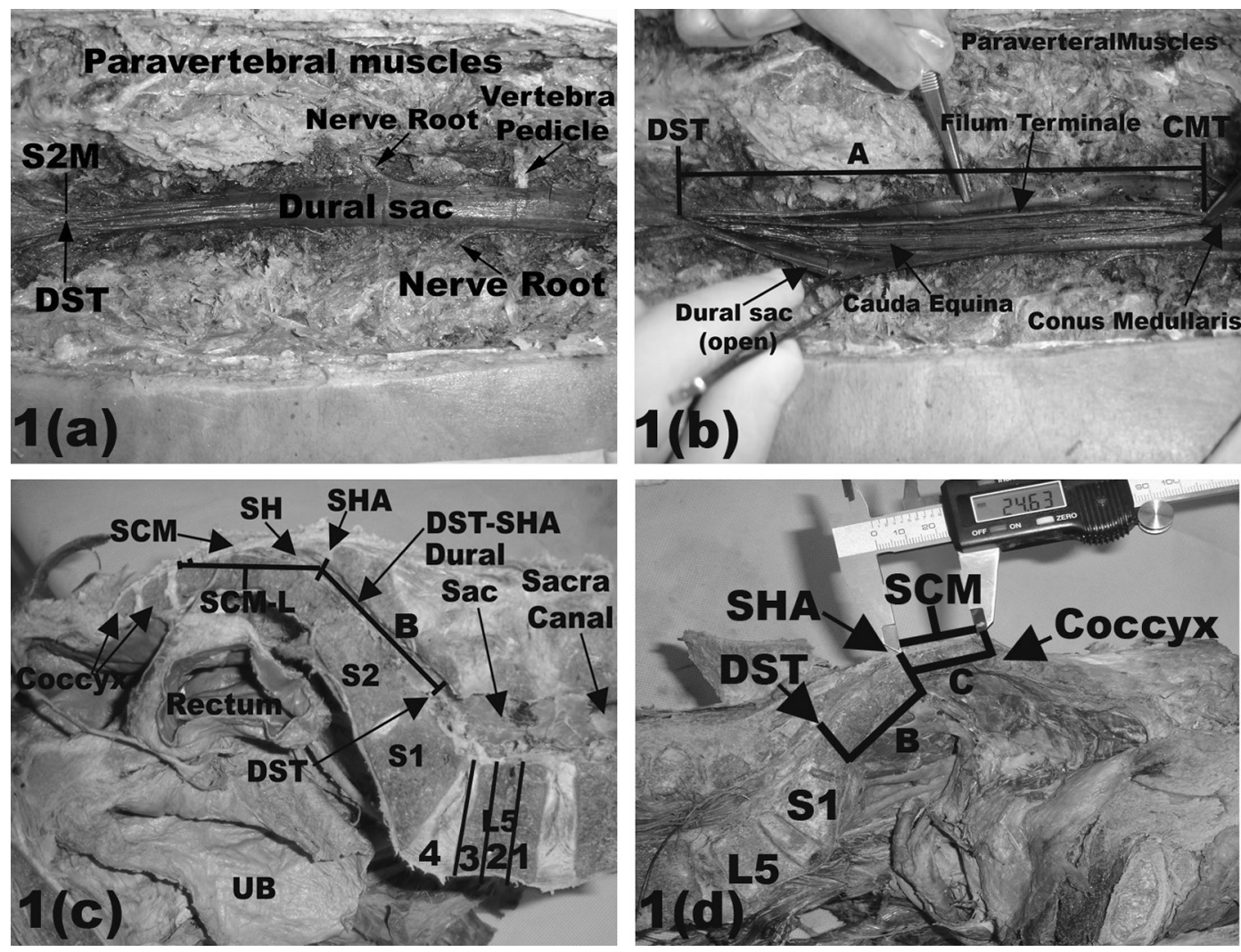

Figure 1. Photographs of human cadaveric specimens showing in Panel a a closed dural sac within the spinal canal after resection of the vertebral laminae with the spinal nerves originating from its sides. Its termination (DST) is seen at the level of middle third of second sacral vertebra (S2M). In Panel $\mathbf{b}$ the opened dural sac shows the method of measuring the linear distance (line A) between the caudal termination of conus medullaris (CMT) and dural sac (DST). The dural sac contains the cauda equina and filum terminale. In Panel c the sagittal section at the lumbosacral area shows the position of dural sac within the vertebral canal, the method of measuring the linear distance (line B) between the vertebral level of sacral hiatus apex (SHA) and DST (DST-SHA), the linear distance between SHA and the coccyx (SCM-L) that represents the length of sacrococcygeal membrane (SCM) and the method of division of the vertebra into three thirds and the following disc into a consequence manner $(1,2,3,4)$. In Panel $\mathbf{d}$ the sagittal section of lumbosacral area shows the linear distance between DST and SHA (line B) and the linear distance between SHA and coccyx (line C) that represent the length of SCM; L5 — fifth lumbar vertebra; S1 — first sacral vertebra; $\mathrm{SH}$ - sacral hiatus.

ittal T2- and T1-weighted images on the lumbosacral region by using a single phased array spine coil, with the patient supine. Sagittal images were obtained by using a slice thickness of $4.0 \mathrm{~mm}$ and interslice gap of $1.0 \mathrm{~mm}$. A repetition time (TR) was $400-500$ for T1-weighted and 3000-4000 for T2-wieghted images, echo time (TE): 80/EF, field of view (FOV): $30 \times 22.5 \mathrm{~cm}$, ET: $12 \mathrm{~ms}$, Se: 2, Im: 6. Vertebral levels of CMT, DST and SHA, length and thickness of SCM, and AP diameter of sacral canal at level of SHA were assessed in all MRI in relation to the adjacent vertebrae and disc spaces (Fig. 2).

In both cadaveric specimens and MRI, each vertebra was divided into three equal parts (upper, middle and lower), and the intervertebral disc space was also considered as one segment. A consecutive numeration was done starting from the up- per segment of T11 vertebra that received number one to the upper segment of $S 5$ vertebra that was numbered 41 to be used as variables. A horizontal line was drawn from the most distal point of conus medullaris, terminal caudal end of dural sac and apex of $\mathrm{SH}$ perpendicular to the longitudinal axis of the spine on the mid-sagittal MRI. The positional levels of CMT, DST and SHA were determined by their corresponding vertebral segment or intervertebral disc space [21].

The linear distance between CMT, DST and SHA were measured. Also, length and thickness of SCM and AP diameter of sacral canal at SHA were measured as well in different age groups of both male and female patients (Fig. 2b). MRI gives wider age range than the cadaveric study and can examine the living subjects, but sometimes the cauda equina can 

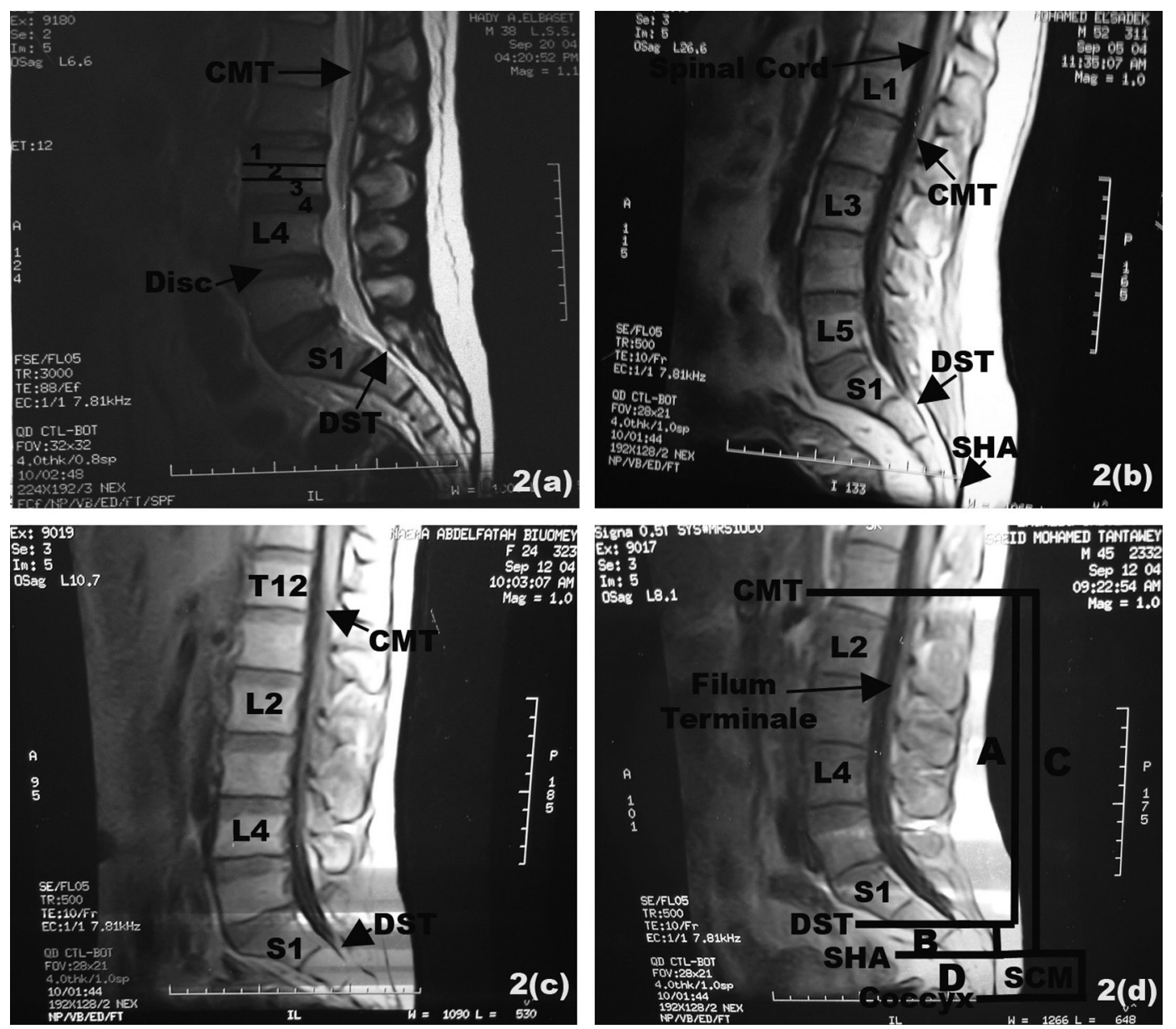

Figure 2. Magnetic resonance of T1- or T2-weighted spin-echo images showing the different levels of conus medullaris termination (CMT), dural sac termination (DST) and sacral hiatus apex (SHA) in relation to their corresponding vertebral segment $(1,2,3)$ or intervertebral disc (4). In Panel d magnetic resonance image shows the method of measuring the linear distance between CMT-DST (line A), DST-SHA (line B), CMT-SHA (line C) and sacrococcygeal membrane (SCM) length (line D).

obscure the image of caudal end of spinal cord and dural sac. In the present study, both cadaveric and MRI studies were used to confirm and compare the obtaining data of each parameter.

\section{Statistical analysis}

Analysis of the data was done by using SPSS software, version 20 for windows (Chicago, III, USA). The mean, median, standard error of the mean (SEM), standard deviation (SD) and the Pearson correlation coefficient, with significance at $p<0.05$ were used. Kolmogorov-Smirnov test was used to test the normality of variables in each group. Mann-Whitney $U$ test, independent T-test and $\chi^{2}$ test were used for comparison of variables between the groups.

This study was performed after the agreement of the medical ethical committee of the faculty of medicine, King Abdulaziz University, Jeddah, Saudi
Arabia and the MRI were taken after the informed consent from the patients.

\section{RESULTS}

The mean age of males was $43.2 \pm 11.9$ and its range was from 16 to 68 years, while that of females was $39.8 \pm 12.1$ with a range of 16 to 65 years.

\section{MRI findings}

The vertebral level of conus medullaris termination (CMT): The mean of the vertebral level of CMT showed lower position (L1-2 disc) in females compared with those of males (L1L) in the corresponding age groups. No significant age or sex difference $(p>0.05)$ in the mean of CMT as it was seen at L1-2 disc in females and at L1L in males in different age groups (Table 1). The range of vertebral level of CMT extended from T12L to L2-3 disc in females and males with no 
Table 1. Vertebral levels of conus medullaris termination, dural sac termination and the sacral hiatus apex at the different age groups of male and female patients using magnetic resonance imaging (mean \pm standard deviation)

\begin{tabular}{|c|c|c|c|c|c|c|}
\hline \multirow[t]{2}{*}{$\begin{array}{l}\text { Age group } \\
\text { [year] }\end{array}$} & \multicolumn{2}{|c|}{$\begin{array}{c}\text { Vertebral level of conus medullaris } \\
\text { termination }\end{array}$} & \multicolumn{2}{|c|}{$\begin{array}{c}\text { Vertebral level of dural sac } \\
\text { termination }\end{array}$} & \multicolumn{2}{|c|}{$\begin{array}{c}\text { Vertebral level of the sacral } \\
\text { hiatus apex }\end{array}$} \\
\hline & Male & Female & Male & Female & Male & Female \\
\hline$<20$ & $\mathrm{~L} 1 \mathrm{~L} \pm 1.6$ & $\mathrm{~L} 1-2 \pm 1.9$ & $\mathrm{~S} 2 \mathrm{U} \pm 1.9$ & $\mathrm{~S} 2 \mathrm{M} \pm 2.0$ & $\mathrm{~S} 4 \mathrm{M} \pm 2.7$ & $S 4 M \pm 1.7$ \\
\hline $20-29$ & $\mathrm{~L} 1 \mathrm{~L} \pm 1.9$ & $\mathrm{~L} 1-2 \pm 1.8$ & $\mathrm{~S} 2 \mathrm{U} \pm 2.3$ & $\mathrm{~S} 2 \mathrm{M} \pm 2.03$ & $S 4 M \pm 2.3$ & $S 4 M \pm 2.5$ \\
\hline 30-39 & $\mathrm{L} 1 \mathrm{~L} \pm 2.0$ & $\mathrm{~L} 1-2 \pm 1.7$ & $\mathrm{~S} 2 \mathrm{U} \pm 1.9$ & $S 2 M \pm 2.2$ & $S 4 M \pm 2.3$ & $S 4 M \pm 2.4$ \\
\hline $40-49$ & $\mathrm{~L} 1 \mathrm{~L} \pm 1.6$ & $\mathrm{~L} 1-2 \pm 2.6$ & $\mathrm{~S} 2 \mathrm{U} \pm 2.02$ & $\mathrm{~S} 2 \mathrm{M} \pm 2.4$ & $S 4 M \pm 2.0$ & $S 4 M \pm 2.2$ \\
\hline $50-59$ & $\mathrm{~L} 1 \mathrm{~L} \pm 1.7$ & $\mathrm{~L} 1-2 \pm 2.04$ & $\mathrm{~S} 2 \mathrm{U} \pm 1.7$ & $\mathrm{~S} 2 \mathrm{M} \pm 1.8$ & $S 4 M \pm 1.95$ & $S 4 M \pm 2.3$ \\
\hline$\geq 60$ & $\mathrm{~L} 1 \mathrm{~L} \pm 1.8$ & $\mathrm{~L} 1-2 \pm 1.4$ & $\mathrm{~S} 2 \mathrm{U} \pm 1.5$ & $\mathrm{~S} 2 \mathrm{M} \pm 1.8$ & $S 4 M \pm 1.8$ & $S 4 M \pm 2.3$ \\
\hline
\end{tabular}

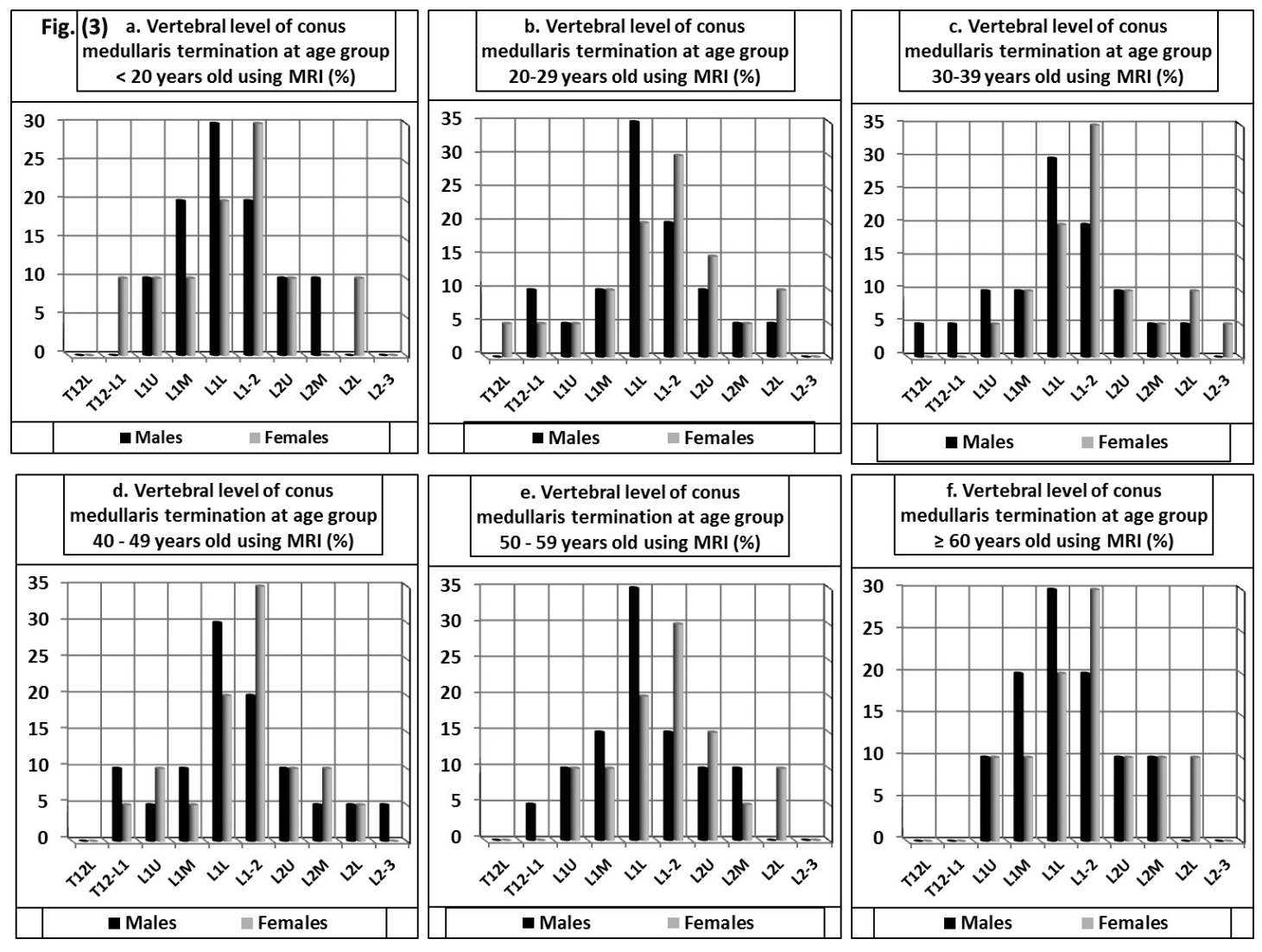

Figure 3. The frequency of the vertebral level of conus medullaris termination at the different age groups in both male and female patients using magnetic resonance imaging (MRI) (\%).

significant difference $(p<0.0001)$. The distribution of CMT showed the highest incidence of CMT (32\% and $35 \%$ ) at the level of $\mathrm{L} 1 \mathrm{~L}$ in males and at $\mathrm{L} 1-2$ disc in females. Also, $51-54 \%$ of cases were seen at the range of $L 1 L$ to $L 1-2$ disc in both males and females, respectively. However, only $1-2 \%$ of cases were seen at a vertebral level below $L 2$ vertebra in males and females (Fig. 3).

The vertebral level of the caudal termination of dural sac (DST): The mean of DST was seen at the upper one-third of S2 (S2U) in males and at middle one-third of S2 (S2M) in females in the different age groups. No significant differences $(p>0.05)$ were observed in the mean of DST between males and females with increasing age (Table 1). The range of DST extended from L5-S1 disc space to the middle one-third of S3 (S3M). The common frequent position of DST level was S2M (32\%) in females and S2U $(30 \%)$ in males. Moreover, in $51 \%$ of cases, the dural sac terminated at the range of vertebral level S2U to S2M in both males and females without sex difference. However, the position of DST was observed in 

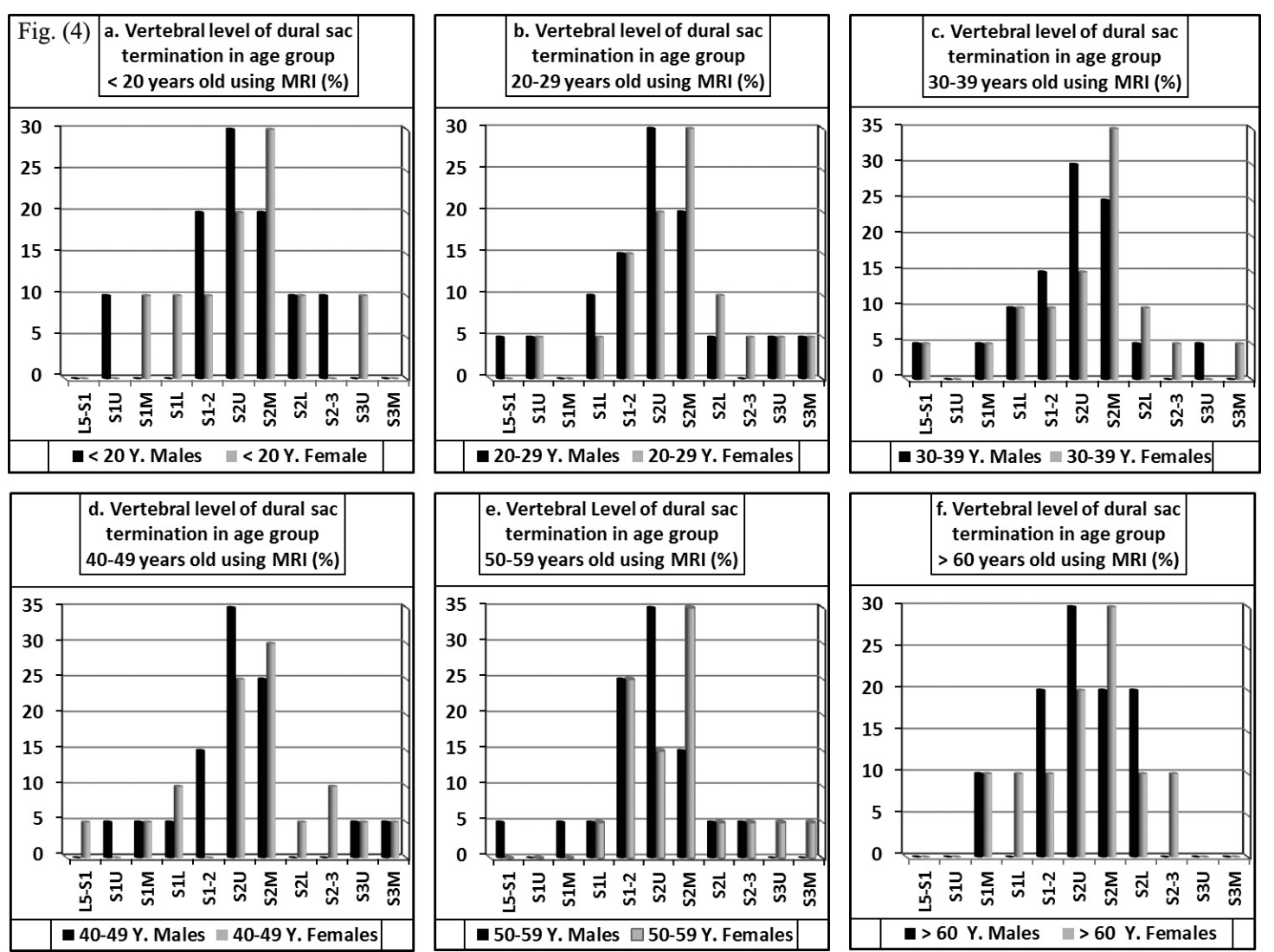

Figure 4. The frequency of the vertebral level of dural sac termination at the different age groups in both male and female patients using magnetic resonance imaging (MRI) (\%).

$7 \%$ on men and $14 \%$ of women below S2 vertebra. Meanwhile, in $3 \%$ of males and $2 \%$ of females was seen at a vertebral level above S1 vertebra. No case was observed below $\mathrm{S} 3$ vertebra in both male and female patients (Fig. 4).

The vertebral level of sacral hiatus apex (SHA): The mean of SHA was seen at the middle one-third of fourth sacral vertebra (S4M) in both male and female patients without changes with increasing age (Table 1). Distribution of the vertebral level of SHA showed $78 \%$ of male and $79 \%$ of female case at vertebral level below S3-4, where $13 \%$ of males and $14 \%$ of female patients was seen at vertebral level S3-4. Only $1 \%$ was seen above S3 (Fig. 5).

MRI measurements of male patients: Mean of the linear distance between CMT and DST (CMT-DST) was $154.3 \pm 4.8 \mathrm{~mm}$ at group of age $<20$ years, $170.2 \pm 5.0 \mathrm{~mm}$ at group of age $30-39$ years and $162.5 \pm 8.4 \mathrm{~mm}$ at group of age $\geq 60$ years. For all male patients, the mean of CMT-DST was $163.9 \pm$ $\pm 8.3 \mathrm{~mm}$. No significant difference $(p=0.47-0.95)$ was reported between the age groups from 20-29 years to $\leq 60$ years regarding the mean linear distance of
CMT-DST, while a significant difference $(p=0.004)$ was recorded in the mean at the age group $<20$ years compared with that of 20-29 years' group. However, regarding the mean distance between DST and SHA (DST-SHA), it measured $52.7 \pm 11.5 \mathrm{~mm}$ at the age group $<20$ years, $56.6 \pm 5.9 \mathrm{~mm}$ at the age group $30-39$ years and $52.3 \pm 5.5 \mathrm{~mm}$ at the age group $\geq 60$ years with no significant difference $(p=0.15-0.91)$ in the mean of DST-SHA distance by increasing age. For all male cases, the mean was $53.6 \pm 7.6 \mathrm{~mm}$. On the other hand, the mean of the distance between CMT and SHA (CMT-SHA) was $196.8 \pm 6.2$ at age group $<20$ years and became $216.9 \pm 7.6 \mathrm{~mm}$ at age group 30-39 years then it measured $204.6 \pm 8.2 \mathrm{~mm}$ at age group $\leq 60$ years. But for all male patients, it measured $210.1 \pm 8.1 \mathrm{~mm}$ with no significant difference $(p=0.06-0.35)$ between the different age groups except first age group $(<20$ years) that revealed a significant difference $(p<0.001)$ compared with that of the second age group (Table 2).

Moreover, the mean of sacrococcygeal membrane length (SCM-L) measured $34.5 \pm 6.8 \mathrm{~mm}$ and that of its thickness (SCM-T) was $3.4 \pm 0.5 \mathrm{~mm}$ in the age 

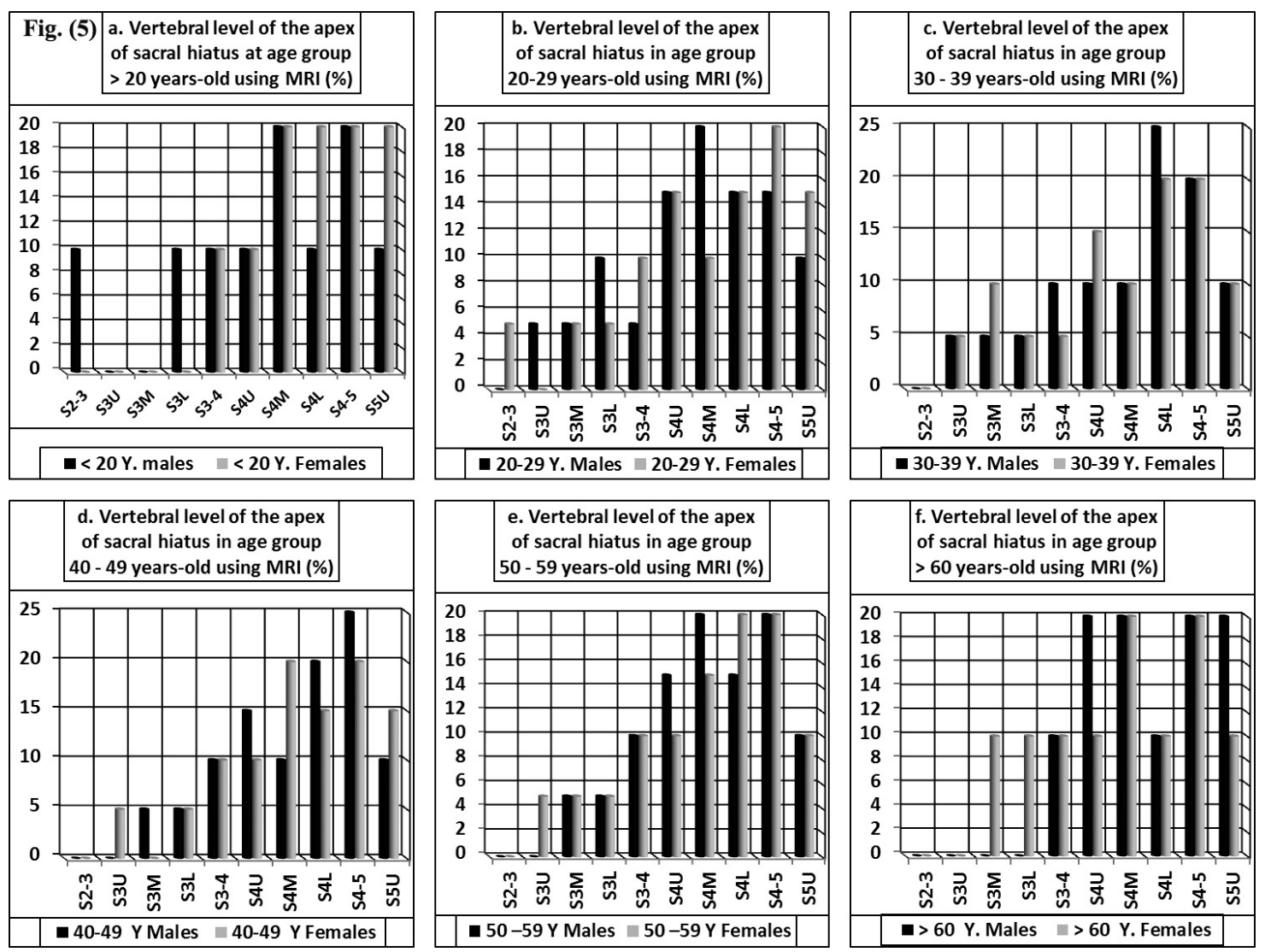

Figure 5. The frequency of the vertebral level of sacral hiatus apex at the different age groups in both male and female patients using magnetic resonance imaging (MRI) (\%).

Table 2. Magnetic resonance imaging measurements of male patients at different age groups (mean \pm standard deviation)

\begin{tabular}{lcccccc}
\hline Age [year] & CMT-DST & DST-SHA & CMT-SHA & SCM-L & SCM-T & SHA-D \\
\hline$<20$ & $154.3 \pm 4.8^{* * *}$ & $52.7 \pm 11.5$ & $196.8 \pm 6.2$ & $34.5 \pm 6.8$ & $3.4 \pm 0.5$ & $5.2 \pm 1.6$ \\
$20-29$ & $167.9 \pm 13.2$ & $53.3 \pm 8.0$ & $212.9 \pm 7.9$ & $35.8 \pm 9.6$ & $3.6 \pm 0.9$ & $5.3 \pm 1.5$ \\
$30-39$ & $170.2 \pm 5.0$ & $56.6 \pm 5.9$ & $216.9 \pm 7.6$ & $37.4 \pm 10.7$ & $3.4 \pm 0.6$ & $5.3 \pm 1.3$ \\
$40-49$ & $165.03 \pm 13.5$ & $54.2 \pm 7.8$ & $214.7 \pm 5.8$ & $38.5 \pm 12.8$ & $3.7 \pm 1.2$ & $5.2 \pm 1.4$ \\
$50-59$ & $163.3 \pm 4.6$ & $52.6 \pm 7.0$ & $208.8 \pm 12.6$ & $37.8 \pm 11.3$ & $3.5 \pm 0.9$ & $5.0 \pm 1.4$ \\
$\geq 60$ & $162.5 \pm 8.4$ & $52.3 \pm 5.5$ & $204.6 \pm 8.2$ & $36.9 \pm 13.7$ & $3.3 \pm 0.8$ & $4.7^{*} \pm 1.4$ \\
All males & $163.9 \pm 8.3^{* *}$ & $53.6 \pm 7.6^{*}$ & $210.1 \pm 8.1^{*}$ & $36.8 \pm 10.8^{*}$ & $3.5 \pm 0.8^{\mathbf{n}}$ & $5.1 \pm 1.4^{\mathbf{n}}$ \\
\hline
\end{tabular}

CMT-DST — distance between conus medullaris termination (CMT) and dural sac termination (DST); SHA-DST — distance between sacral hiatus apex (SHA) and DST; CMT-SHA — distance between CMT and SHA; SCM-L — length of sacrococcygeal membrane (SCM); SCMT — thickness of SCM; SHA-D — anteroposterior diameter of sacral canal at SHA; ${ }^{*} p<0.0001 ;{ }^{* *} p=0.0149 ;{ }^{* * *} p=0.004$ compared with age group of $20-29$ years; $n-$ non-significant $(p=0.63,0.26)$ compared with the corresponding measures of female patients

group of $<20$ years; then the mean of SCM-L was $36.9 \pm 13.7 \mathrm{~mm}$ and that of SCM-T measured $3.3 \pm$ $\pm 0.8 \mathrm{~mm}$ in the age group of $\geq 60$ years. The mean of SCM-L for all male patients was $36.8 \pm 10.8 \mathrm{~mm}$ and that of SCM-T measured $3.5 \pm 0.8 \mathrm{~mm}$. No significant age difference $(p=0.62-0.86)$ was recorded at the mean of SCM-L and SCM-T by increasing age. In addition, the mean of the AP diameter of sacral canal at the apex of sacral hiatus (SHA-D) was $5.2 \pm$ 1.6 at age group of $<20$ years, $5.3 \pm 1.3 \mathrm{~mm}$ at age group of 30-39 years; then it became $4.7 \pm 1.4 \mathrm{~mm}$ at the age group of $\geq 60$ years, while that of all male patient measured $5.1 \pm 1.4 \mathrm{~mm}$ with no significant difference ( $p>0.5$ ) with increasing age (Table 2 ).

MRI measurements of female patients: The mean of the distance between CMT-DST measured $151.6 \pm 7.8 \mathrm{~mm}$ in subjects $<20$ years, $165.5 \pm 10.8 \mathrm{~mm}$ in subjects aged $30-39$ years, and then it became $161.5 \pm 8.7 \mathrm{~mm}$ in the age group of $\geq 60$ years, while the mean of CMT-DST linear distance of all female patients was $161.2 \pm 7.2 \mathrm{~mm}$. No significant differences $(p=0.28-0.83)$ in the mean of CMT-DST 
Table 3. Magnetic resonance imaging measurements of female patients at different age groups (mean \pm standard deviation)

\begin{tabular}{lcccccc}
\hline Age [year] & CMT-DST & DST-SHA & CMT-SHA & SCM-L & SCM-T & SHA-D \\
\hline$<20$ & $151.6 \pm 7.8^{*}$ & $45.2 \pm 5.2$ & $186.6 \pm 9.4^{*}$ & $30.8 \pm 7.6$ & $3.5 \pm 0.3$ & $5.3 \pm 1.5$ \\
$20-29$ & $162.4 \pm 6.5$ & $47.8 \pm 10.0$ & $209.9 \pm 6.4$ & $32.5 \pm 6.8$ & $3.6 \pm 0.2$ & $5.5 \pm 1.4$ \\
$30-39$ & $165.5 \pm 10.8$ & $50.7 \pm 10.5$ & $205.7 \pm 8.2$ & $33.2 \pm 10.1$ & $3.8 \pm 0.4$ & $5.4 \pm 1.4$ \\
$40-49$ & $163.3 \pm 4.6$ & $48.3 \pm 8.7$ & $201.2 \pm 7.3$ & $34.3 \pm 8.7$ & $3.6 \pm 0.7$ & $5.2 \pm 1.6$ \\
$50-59$ & $162.6 \pm 13.9$ & $46.5 \pm 15.2$ & $204.9 \pm 8.4$ & $32.6 \pm 8.0$ & $3.5 \pm 0.6$ & $5.2 \pm 1.6$ \\
$\geq 60$ & $161.5 \pm 8.7$ & $45.7 \pm 10.2$ & $198.5 \pm 9.7$ & $32.1 \pm 6.1$ & $3.4 \pm 0.3$ & $4.9 \pm 1.5$ \\
All females & $161.2 \pm 7.2$ & $47.4 \pm 9.9$ & $201.1 \pm 8.2$ & $32.5 \pm 7.9$ & $3.6 \pm 0.4$ & $5.2 \pm 1.5$ \\
\hline
\end{tabular}

CMT-DST — distance between conus medullaris termination (CMT) and dural sac termination (DST); SHA-DST — distance between sacral hiatus apex (SHA) and DST; CMT-SHA — distance between CMT and SHA; SCM-L — length of sacrococcygeal membrane (SCM); SCM-T — thickness of SCM; SHA-D — anteroposterior diameter of sacral canal at SHA; ${ }^{*}$ positive significant difference of the measurements of the age group of $<20$ years compared with that of the age group of 20-29 years $(p=0.004)$

distance comparing the different age groups from 20-29 years to $\leq 60$ years, while the mean of first age group of $<20$ years revealed a significant difference $(p=0.004)$ compared with that of second age group (20-29 years). Moreover, the mean of the distance between DST and SHA (DST-SHA) increased from $45.2 \pm 5.2 \mathrm{~mm}$ in the age group of $<20$ years to $50.7 \pm 10.5 \mathrm{~mm}$ in age group of 30-39 years, then it became $45.7 \pm 10.2 \mathrm{~mm}$ in age group of $\geq 60$ years. The mean of the DST-SHA distance for all female cases was $47.4 \pm 9.9 \mathrm{~mm}$. The mean of DST-SHA distance revealed no significant difference with increasing age $(p=0.28-0.88)$. Also, the mean distance between CMT-SHA measured $186.6 \pm 9.4 \mathrm{~mm}$ in the age group of $<20$ years, $205.7 \pm 8.2 \mathrm{~mm}$ in the age group of $30-39$ years and $198.5 \pm 9.7 \mathrm{~mm}$ in the age group of $\geq 60$ years, while the mean of all female cases was $201.1 \pm 8.2 \mathrm{~mm}$. The mean of CMT-SHA distance at the age group of $<20$ years showed a significant $(p<0.001)$ difference compared with that of second age group (20-29 years), otherwise no significant difference ( $p=0.072-0.15$ ) was recorded with increasing age (Table 3 ).

In addition, the mean of SCM-L of female patients was $30.8 \pm 7.6 \mathrm{~mm}$ in the age group of $<20$ years, $33.2 \pm 10.1 \mathrm{~mm}$ in the age group of 30-39 years and $32.1 \pm 6.1 \mathrm{~mm}$ in the age group of $\geq 60$ years. The mean of this distance for all female cases was $32.5 \pm$ $\pm 7.9 \mathrm{~mm}$ with no significant age difference $(p=$ $=0.52-0.86$ ). The mean of SCM-L for all female patients was $32.5 \pm 7.9 \mathrm{~mm}$. Meanwhile, the mean of SCM-T measured $3.5 \pm 0.3 \mathrm{~mm}$ in the age group of $<20$ years and $3.4 \pm 0.3 \mathrm{~mm}$ in the age group of $\geq 60$ years with no age significant difference $(p>0.05)$. Moreover, the mean AP diameter of $\mathrm{SH}$ at SHA was $5.3 \pm 1.5 \mathrm{~mm}$ in the age group of $<20$ years, $5.4 \pm$ $\pm 1.4 \mathrm{~mm}$ in the age group of 30-39 years, $4.9 \pm$ $\pm 1.5 \mathrm{~mm}$ in the age group of $\geq 60$ years and it measured $5.2 \pm 1.5 \mathrm{~mm}$ for all female cases. The mean of all male patients revealed significant differences $(p<0.05)$ compared with that of female patients in CMT-DST distance, DST-SHA distance and CMT-SHA distance and length of SCM (Table 3).

\section{Findings of the cadavers}

In cadaveric specimens, no significant difference ( $p>0.05$ ) was recorded in the vertebral level of CMT, DST and SHA in male compared with those of female cadavers, where the mean of the vertebral level of CMT was seen at L1L, that of DST at S2M and that of SHA at S4U in both male and female cadavers (Table 4). Moreover, the mean of the distance between CMT and DST measured $164.8 \pm 26.6 \mathrm{~mm}$ in male and $141 \pm 19.9 \mathrm{~mm}$ in female cadavers $(p<0.005)$, while the mean of the distance between DST and SHA was $50.6 \pm 10.6 \mathrm{~mm}$ in male and $48.6 \pm 8.9 \mathrm{~mm}$ in female cadavers $(p=0.47)$ and the mean linear distance between CMT and SHA measured $227.9 \pm 25.7 \mathrm{~mm}$ in male and $215.2 \pm 19.9 \mathrm{~mm}$ in female cadavers $(p=0.058)$. Meanwhile, no significant difference $(p>0.05)$ was reported in SCM-L and SCM-T of male cadavers compared with those of female cadavers, where the mean of SCM-L measured $35.2 \pm 8.2 \mathrm{~mm}$ in male cadavers and that of female cadavers was $32.3 \pm 7.6 \mathrm{~mm}(p=0.19)$. Moreover, the mean of SCM-T in male cadavers was $3.25 \pm 0.7 \mathrm{~mm}$ and that of female cadavers was $3.48 \pm 0.9 \mathrm{~mm}(p=0.26)$. Moreover, the mean of AP diameter of the sacral canal at the level of SHA was $4.8 \pm 1.5 \mathrm{~mm}$ in male cadavers, while that of female cadavers measured $5.1 \pm 1.6 \mathrm{~mm}$ with no significant difference $(p=0.44)$ in-between. The AP diameter less than $2 \mathrm{~mm}$ was seen in 1 (2.5\%) male and 1 (5\%) female cadaver. 
Table 4. Vertebral levels and measurements of conus medullaris termination, dural sac termination and sacral hiatus apex in male and female cadavers (mean \pm standard deviation [SD])

\begin{tabular}{|c|c|c|c|c|c|c|}
\hline \multirow{2}{*}{$\begin{array}{l}\text { Vertebral levels and } \\
\text { measurements }\end{array}$} & \multicolumn{2}{|c|}{ Male cadavers (40) } & \multicolumn{2}{|c|}{ Female cadavers $(20)$} & \multirow[t]{2}{*}{ t-value } & \multirow[t]{2}{*}{ p-value } \\
\hline & Mean \pm SD & Range & Mean \pm SD & Range & & \\
\hline CMT & $\mathrm{L} 1 \mathrm{~L} \pm 1.91$ & T12L-L2-3 & $\mathrm{L} 1 \mathrm{~L} \pm 1.87$ & T12L-L2-3 & 0.35 & 0.73 \\
\hline DST & $\mathrm{S} 2 \mathrm{M} \pm 1.9$ & S1U-S3M & $\mathrm{S} 2 \mathrm{M} \pm 1.95$ & S1U-S3M & 1.96 & 0.055 \\
\hline SHA & S4U \pm 2.04 & S3L-S5U & S4U \pm 1.39 & S3L-S5U & 0.17 & 0.87 \\
\hline AP diameter at SHA & $4.8 \pm 1.5$ & $1.76-8.29$ & $5.1 \pm 1.6$ & $1.86-8.78$ & 0.79 & 0.44 \\
\hline CMT-DST distance & $164.8 \pm 26.6$ & $113.5-201.3$ & $140.95 \pm 19.9$ & $106.5-175.9$ & 3.54 & $0.0008^{*}$ \\
\hline DST-SHA distance & $50.6 \pm 10.6$ & $32.2-72.4$ & $48.6 \pm 8.9$ & $35.9-65.9$ & 0.73 & 0.47 \\
\hline CMT-SHA distance & $227.9 \pm 25.7$ & $175.8-260.5$ & $215.2 \pm 19.9$ & $173.7-240.2$ & 1.94 & 0.058 \\
\hline SCM-L & $35.2 \pm 8.2$ & $20.2-54.2$ & $32.3 \pm 7.6$ & $21.7-50.3$ & 1.3 & 0.19 \\
\hline SCM-T & $3.25 \pm 0.7$ & $2.15-4.92$ & $3.48 \pm 0.9$ & $2.21-5.32$ & 1.14 & 0.26 \\
\hline
\end{tabular}

CMT — conus medullaris termination; DST — dural sac termination; SHA — sacral hiatus apex; AP diameter at SHA — anteroposterior diameter at SHA; CMT-DST — distance between CMT and DST; SHA-DST — distance between SHA and DST; CMT-SHA — distance between CMT and SHA; SCM-L — length of sacrococcygeal membrane (SCM); SCMT-T — thickness of SCM; * positive significant difference between male and female cadavers at $p=0.0008$

\section{DISCUSSION}

Knowledge of the exact position of CMT, DST and SHA levels is essential before doing lumber puncture or caudal injection to avoid the iatrogenic injury of spinal cord or the roots of cauda equina. MRI which is non-invasive procedure gives the chance to evaluate the distribution of the CMT, DST and SHA in the living populations at different age and sex groups. MRI findings of the present study revealed that, the mean of CMT was L1-2 in female and L1L in male patients, while its range extended from $T 12 \mathrm{~L}$ to $\mathrm{L} 2-3$ but with no sex or age difference ( $p>0.05)$. Also, the mean of CMT in cadaveric specimens was seen at L1 L in both male and female cadavers. In disagreement with the findings of this study, a positive correlation between CMT and increasing age has reported in previous studies [4, 25]. Demiryürek et al. [4] added that, the position of CMT was most commonly observed at T12-L1 intervertebral disc space in the whole population, while Soleiman et al. [25] stated that, the mean of CMT was at the level of the middle one-third of L1 (L1M) and its range span extended from the lower one-third of T11 (T11L) to the upper one-third of L3 (L3U) but with a small and significant positive correlation between CMT position and age. Also, in contradiction to the results of this study, Lirk et al. [8] stated that older people in general showed a lower CMT position than younger people. The caudal level of CMT in old age might be due to the degenerative changes of spine that could be occurred with increasing age, not due to the age per se [25].

Similar to the results of the present study, no significant difference in the vertebral level of CMT was recorded with increasing age as the mean of conus site was located at the level of lower one-third of L1 (L1L) and its range extended from the middle one-third of $T 12$ to the upper one-third of $L 3$ using sagittal MRI of adult 273 females and 231 males [21]. Moreover, Kim et al. [5] in healthy adults reported that, the mean of CMT position was located at the lower one-third of L1. However, their results did not correlate with the findings of the present study regarding the range of CMT. This difference might be related to the number of cases used in each study. The position of CMT at L2-L3 or above should be considered normal at any age, while the conus at L3 or below was considered as an indeterminate normal or tethered conus at this level [28]. In the present results, no case showed vertebral level of CMT below L2-3 disc space.

In other studies, the terminal position of the conus medullaris in children reached the adult level (L1-L2) by 2 years of age [21]. Moreover, the later author added that, the range of CMT for the $0-2$-year-old group and 19-20-year-old group was the same at T12 to $L 2-L 3$, and the mean of their CMT level was L1-2 space at the two age groups. The conus medullaris attained the adult level sometime during the first few months of life without changes throughout childhood as stated by Wilson and Prince [28].

The effect of sex on the level of CMT remains controversial, although many studies have been done. In the results of present study, no significant sex difference ( $p>0.05$ ) was reported in the mean of the vertebral level of CMT position as it was seen at L1-2 disc space in female patients and at L1L in male patients by using MRI. Also, in cadaveric specimens, the mean of CMT position was observed at L1L in both male and female cadavers. These findings were con- 
sistent with those of Saifuddin et al. [21], Macdonald et al. [9], Demiryurek et al. [4] and Moussallem et al. [12] who concluded that, the lower position of CMT in females was insignificant compared with those of males. In agreement with the results of the previous studies, Rahmani et al. [19] found insignificant sex difference regarding the level of CMT that was seen at L1 middle one-third in females and at L1 upper onethird in males. In addition, Kim et al. [5], observed no difference in spinal cord length based on sex in the general population and in absence of disease states.

However, Kim et al. [5] reported that, the mean of CMT was at L1 lower one-third (L1L) in male, at L1-2 disc space in female and at L1 L in all with a sex significant difference in age groups of more than 50 years. Thus, on the basis of the findings of the present study, the effect of sex alone on the spinal cord length seemed to be uncertain, however the lower position of CMT in females increased its susceptibility to the iatrogenic injuries than in males. Meanwhile, Lin et al. [7] postulated that, sex might affect the CMT position in various disease states, and hence could be an important factor, where analysis between males and females with bulging discs, lumbar intervertebral disc herniation and thoracic vertebral compression fractures displayed a significant difference, with females having a lower CMT position in each case. Thus, the effect of sex alone on spinal cord length was uncertain. However, CMT position in females was more susceptible to the effects of spinal disease than in males [7]. In disagreement with the results of this study, a significant difference was found between CMT position in females and males [25]. Soleiman et al. [25] reported that, the mean of CMT position was noted at L1M in females and at L1L in males, while Demiryurek et al. [4] found a significant sex difference in the mean of CMT level in Turkish, where in males it was found at the level of T12-L1 disc space, while in females it was seen at L1-2 disc space. The race of the population might have a role in the sex difference of the position of CMT.

In literature, the caudal position of CMT has been determined in cadavers [11, 15, 20]. Mc Cotter [11], in 234 cases, reported that there was no relation between cord length and vertebral column. In his series the level of the CMT varied between the middle of $\mathrm{T} 12$ and the lower border of $\mathrm{L} 2$, but in $77 \%$ of white males the cord termination was between the upper border of L1 and L2 [11]. Moreover, Needles [15], reported that the cord termination was between the middle one-third of T12 and the lower one-third of L3. In $49 \%$ of his series the CMT level was between the lower one-third of $\mathrm{L} 1$ and the upper one-third of L2 [15]. Reiman and Anson [20], noticed the mean conus position at $\mathrm{L} 1-\mathrm{L} 2$, ranging between the lower one-third of $\mathrm{T} 12$ and the middle one-third of $\mathrm{L} 3$ among 198 cadavers with a lower position of CMT in females than in males. In the findings of present study, the mean of CMT was L1L in both male and female cadavers [20].

Knowledge of the correlation between position of CMT and DST is important in the practice of spinal and caudal anaesthesia. The results of the present study revealed that, the mean level of DST was seen at the upper one-third of $\mathrm{S} 2(\mathrm{~S} 2 \mathrm{U})$ in males and at middle one-third of S2 (S2M) in females and its range extended from L5-S1 disc space to S3M across the all age groups using MRI. In accordance with the data of this study, Macdonald et al. [9] found a minor sex difference in the mean of DST, at S2U in men and at S2M in females. However, other studies on the lumbar spine utilising MRI to visualise the DST demonstrated that the mean level of DST was seen at S2M with no significant differences between males and females $[2,3,5,12]$, although it differed from the previous studies where the mean level of DST was reported at S2U $[22,25]$ and at S1-2 [17]. The ethnic differences, age and sample size might be relevant to the diverse findings of these studies.

In the present study, the commonest incidence of DST (30-35\%) was observed at S2U in male and at S2M in female patients; while in $5 \%$ of male and $8 \%$ of female patients, the DST was seen below S2-3 disc with no case below S3. However, in the previous studies higher values of DST were found at a vertebral level below S2-3 disc; in $8 \%$ at S3 [1]; in $8.7 \%$ [22] and in $13.9 \%$ [2]. Consequently, these cases would be undertreated if the lower border of the craniospinal irradiation spinal field were placed at the inferior border of $\mathbf{S} 2$ with negative effects on local control of the disease [17]. Moreover, the later authors added that, the caudal extension of DST lower than the expected sacral level might increase the risk of dural puncture or inadvertent spinal anaesthesia dung caudal epidural block.

In agreement with the results of the present study, Scharf et al. [22] found the upper limit of DST range at L5-S1 disc space, while a higher level was reported at lower one-third of $L 5[1,17]$ and at lower one-third of L3 [25], but a lower level of the upper limit of DST 
range was reported at upper one-third of $\mathrm{S} 1[5,9,12]$. Regarding the lower limit of the range of DST, it was noticed at S3M in the current study, similar findings were reported by Phongkitkarun et al. [17]. However, a higher level at S3U [22] and a lower levels at S3L $[2,5]$; at S3-4 [12]; at S4U [9]; at S5U [25] were previously reported in literatures. The data of this study revealed a relatively narrower range than that previously reported by Macdonald et al. [9], Soleiman et al. [25], Cilliers et al. [2], Moussallem et al. [12] who found the lowest end of the dural sac at the S3-4 interspace, S4 body, or S4-5 interspace. On the basis of the above information, the patients with range spectrum of DST could receive unnecessary irradiation to a critical structure especially the ovaries or testes if the lower border of the craniospinal irradiation spinal field was placed at the lower border of S2 [17].

In accordance with the results of this study, no significant differences in the levels of DST were observed with increasing age $[2,5,10,12]$. However, in other studies an elevation in the level of DST was observed with increasing age especially after 50 years $[17,25]$.

To the best of our knowledge, no previous literature studied the vertebral level of CMT and DST with special reference to the level of SHA in both cadavers and MRI. In the present study, the mean of vertebral level of SHA was S4M in both females and males without changes with increasing age with an incidence of $78 \%$ below S3-4 disc space and only $1 \%$ above S3. The commonest vertebral level of SHA was $\$ 4$ including S4-5 disc space in male $(66 \%)$ and female $(64 \%)$ cases, while in $13 \%$ of cases SHA was found at level of S3. Only $1 \%$ was seen at the level above $\mathrm{S} 3$ vertebra in both males and females. The frequent distribution of SHA revealed no sex difference as well. Various studies have reported similar results namely Nagar [14] in 55.9\% and Sekiguchi et al. [23] in $64 \%$ cases at the level of $\$ 4$. However, the level of S3 was the most common location of SHA in AP lumbosacral spine radiographs in total, males, and females $(48.3 \%, 46.7 \%$, and $50 \%$, respectively [13]. The later author added that, high level of SHA at $\mathrm{S} 3$ or above was considered as a dangerous site, because of its close relation to the level of DST at S2. In the present results, $12 \%$ of men and $13 \%$ of female cases showed SHA at the level of S3.

The sagittal AP diameter at SHA is the narrowest part of the hiatus and it is one of the most important parameters for the success of CEB, thus it should be sufficiently wide to admit a 22 gauge $(0.643 \mathrm{~mm}$ di- ameter) needle [23]. In agreement with the findings of this study, the mean value of AP diameter of the sacral canal at the SHA was $5 \mathrm{~mm}$ [23], and $5.03 \pm 1.3 \mathrm{~mm}$ [1] as the mean of AP diameter at SHA measured $5.24 \mathrm{~mm}$ in female and 5.21 in male patients using MRI and it was $5.1 \pm 1.6 \mathrm{~mm}$ in female and $4.8 \pm 1.5 \mathrm{~mm}$ in male cadavers in the present study. However, in other studies lower values of the mean of AP diameter at SHA was reported $4.46 \pm 1.33 \mathrm{~mm}$ [24] and 4.88 $\mathrm{mm}$ [14]. These values were in agreement with those reported by Mustafa et al. [13] who stated that the mean value of AP diameter of sacral canal at SHA was $4.8 \pm 1.9,5.5 \pm 1.97$, and $4.1 \pm 1.64 \mathrm{~mm}$ in total, male and female sacra, respectively. However, if the AP diameter of sacral hiatus was less than $2 \mathrm{~mm}$, it might cause difficulties and failure in CEB [24]. Variable incidence of the AP diameter of sacral canal at SHA below $2 \mathrm{~mm}$ was reported 15.6\% [14], 6.25\% [24], 7.7\% [27] and in only $1 \%$ of sacral bones [23]. The difference in the results of the previous studies might be attributed to racial diversity, sample size, age effect, or type of research on dry bones, cadavers or by using MRI.

Awareness of the level at which the conus medullaris and dural sac termination and their distances from the $\mathrm{SH}$ is extremely important for anaesthesiologists. As it well increase their confidence to perform CEB as well as decrease the risk of possible complications. In the present study, mean linear distance between CMT and DST measured $163.9 \pm 8.9(126-194.3) \mathrm{mm}$ in males; $161.2 \pm 7.2(112.6-187.3) \mathrm{mm}$ in females using MRI; $164.8 \pm 26.6$ (113.5-201.3) in male cadavers and $141 \pm 19.9$ (106.5-175.9) $\mathrm{mm}$ in female cadavers with no age, but with sex significant difference. Similar to the findings of the present study, Pinto et al. [18] reported that the mean of the intrathecal part of filum terminale, that equivalent to CMT-DST distance, was $154.4 \pm 21.3(112.8-211.1) \mathrm{mm}$ in human cadavers. Moreover, Moussallem et al. [12] stated that the distance between CMT and DST ranged from 5 to 7 vertebral segments with no correlation between age, sex and CMT or DST [12]. However, Matveeva et al. [10] found that the distance between CMT and DST was $23.7 \pm 2.25(18-29)$ vertebral segments. These segments were equivalent to $4.5-7$ units of the vertebral body and intervertebral discs [10].

The caudal space is an epidural space found between the termination of the dural sac and the termination of the sacral canal [26]. In the results of the present study, the mean of the linear distance 
between DST and SHA was $53.6 \pm 7.6(32.4-70) \mathrm{mm}$ in males and $47.4 \pm 9.9(30.2-78.6) \mathrm{mm}$ in females using MRI with a positive sex significant difference $(p<0.05)$. In agreement with the results of the present study, the mean of the distance between DST and SHA measured $45 \mathrm{~mm}$ [27]. Moreover, Mustafa et al. [13] reported that, the main distance between the S2 foramen and SHA was $41 \pm 11.4 \mathrm{~mm}, 36.7 \pm$ $\pm 12.1 \mathrm{~mm}$, and $44.8 \pm 10.1 \mathrm{~mm}$ in total, female and male sacra, respectively. The later authors added that, the values of male revealed significant increase compared with those of females. So, the needle should be advanced few millimetres $(<5 \mathrm{~mm})$ after penetrating the SCM during CEB in adults $[24,27]$. However, the mean distance between the S2 foramen and SHA was $35.4 \pm 10.4 \mathrm{~mm}$ (range 11-62 $\mathrm{mm}$ ) and the distance to the base of the SH was $65.3 \pm 9.4 \mathrm{~mm}$ (range 39-85 mm) [24]. Similarly, the distance between S2 foramen from one side and SHA and base of SH on the other side measured $32.88 \pm 13.34 \mathrm{~mm}$ (range 17-65 mm) and $67.01 \pm 12.08 \mathrm{~mm}$ (range 41-103 $\mathrm{mm})$, respectively [16].

In the present cadaveric study, the mean of the distance between DST and SHA measured $50.6 \pm$ $\pm 10.6 \mathrm{~mm}(32.2-72.4)$ in male and $48.6 \pm 8.9 \mathrm{~mm}$ (35.9-65.9) in female cadavers; however, a lower values were reported by Aggarwal et al. [1] who stated that, the distance between DST and SHA was $31.9 \pm 11.8 \mathrm{~mm}$ with a range of $5.8-59.96 \mathrm{~mm}$. Meanwhile, higher values were stated by Crighton et al. [3] who found that, the mean distance between DST and SHA was $64.6 \pm 12.8 \mathrm{~mm}(41-78)$ in male, $58.5 \pm 13 \mathrm{~mm}(34-80)$ in female and $60.5 \pm 13.1 \mathrm{~mm}$ (34-80) in all cadavers.

In the present study, no significant difference ( $p=0.19$ ) was recorded in the mean length of SCM in female cadavers compared with that of male cadavers, where the mean length of SCM measured $35.2 \pm 8.2(20.2-54.2) \mathrm{mm}$ in male and $32.3 \pm 7.6$ (21.7-50.3) $\mathrm{mm}$ in female cadavers. However, in the MRI measurements of the present study, a significant difference ( $p=0.0013$ ) was determined between the mean of SCM length of male compared with those of female patients as it was $36.8 \pm 10.8 \mathrm{~mm}$ in male and $32.4 \pm 6.9 \mathrm{~mm}$ in female patients. These measurements were similar to the mean length of $34.3 \pm 7.4$ (18.25-48.96) obtained from a cadaveric study [1]. However, lower values were reported in an earlier cadaveric [6] and MRI [3] studies. The mean length of SCM in the cadaveric specimens was
$26.3 \mathrm{~mm}$ [6]. In agreement with the previous study, the mean of length of SCM was $22.6 \pm 6.8(13-36)$ in female and $22.8 \pm 6.9(11-31) \mathrm{mm}$ in male patients with no significant sex difference [3]. Moreover, the later authors added that, the SCM was thicker in female (3.6 \pm 1 with a range of $1-5 \mathrm{~mm}$ ) than that of male $(2.5 \pm 0.91$ with a range of $1-4 \mathrm{~mm})$ patients $(p=0.005)$. Similar findings were observed in the present study as the mean thickness of SCM was $3.6 \pm 0.4(2-6.1) \mathrm{mm}$ in female and $3.5 \pm 0.8(2.1-6)$ $\mathrm{mm}$ in male patients. Thus, to reduce the frequency of dural puncture and other possible complications, the needle should be cautiously introduced through the base of SH after penetrating the SCM [16].

\section{CONCLUSIONS}

The present study demonstrated that the mean of the vertebral levels of the caudal termination of conus medullaris and dural sac revealed no significant difference between their position and age. However, lower position of both CM and DST were observed in female patients. Thus, when performing lumber puncture, the clinician should be aware of the potential difference in the position of the caudal termination of conus medullaris and dural sac especially in female patients, who may have lower CMT than L2. Also, attention is needed in the cases having higher position of DST as lumber puncture at L2-3 interspace would seem to be not suitable in this patient. The findings of this study could be used as a general guideline for anaesthetists and neurosurgeons who perform any diagnostic or therapeutic interventions within the dural sac or caudal space to avoid the occurrence of any iatrogenic injury to spinal cord or/and cauda equina.

\section{Acknowledgements}

Great appreciations to all technical members of the Anatomy Department, Faculty of Medicine, King Abdulaziz University for their help during doing this work.

\section{REFERENCES}

1. Aggarwal A, Kaur H, Batra YK, Aggarwal AK, Rajeev S, Sahni D (2009) Anatomic consideration of caudal epidural space: a cadaver study. Clin Anat, 22: 730-737.

2. Cilliers A, Schulenburg DH, Janse van Rensburg J (2010) MRI determination of the vertebral termination of the dural sac tip in a South African population: clinical significance during spinal irradiation and caudal anaesthesia. SAJ Radiol, 14: 52-55. 
3. Crighton IM, Barry BP, Hobbs GJ (1997) A study of the anatomy of the caudal space using magnetic resonance imaging. Br J Anaesth, 78: 391-395.

4. Demiryürek D, Aydingöz Ü, Akşit MD, Yener N, Geyik PÖ (2002) MR imaging determination of the normal level of conus medullaris. Clin Imag, 26: 375-377.

5. Kim JT, Bahk JH, Sung J (2003) Influence of age and sex on the position of the conus medullaris and Tuffier's line in adults. Anesthesiology, 99: 1359-1363.

6. Lanier VS, McKnight HE, Trotter M (1944) Caudal analgesia: an experimental and anatomical study. Am J Obstet Gynecol, 47: 633-641.

7. Lin N, Bebawy JF, Hua L, and Wang BG (2010) Is spinal anaesthesia at L2-L3 interspace safe in disorders of the vertebral column? A magnetic resonance imaging study. Br J Anaesthesia, 105: 857-862.

8. Lirk P, Messner H, Deibl M, Mitterschiffthaler G, Colvin J, Steger B, Rieder J, Keller C (2004) Accuracy in estimating the correct intervertebral space level during lumbar, thoracic and cervical epidural anaesthesia. Acta Anaesthesiol Scand 48:347-349.

9. Macdonald A, Chatrath P, Spector T, Ellis H (1999) Level of termination of the spinal cord and the dural sac: a magnetic resonance study. Clin Anat, 12: 149-152.

10. Matveeva Niki, Chabukovska Radulova J, Strateska A, Bojadzieva B, Trpkovska B (2013) Anatomic landmarks for save spinal and epidural anesthesia. Acta Morphol, 10: 10-14.

11. Mc Cotter RE (1916) Regarding the length and extent of the human medulla spinalis. Anat Rec, 10: 559-564.

12. Moussallem CD, El Masri H, El-Yahchouchi C, Abou Fakher F, Ibrahim A (2014) Relationship of the lumbar lordosis angle to the level of termination of the conus medullaris and thecal sac. Anat Res Int, 2014: 351769.

13. Mustafa MS, Mahmoud OM, El Raouf HH, Atef HM (2012) Morphometric study of sacral hiatus in adult human Egyptian sacra: their significance in caudal epidural anesthesia. Saudi J Anaesth, 6: 350-357.

14. Nagar SK (2004) A study of sacral hiatus in dry human sacra. J Anat Soc India, 53: 18-21.

15. Needles JH (1935) The caudal level of termination of the spinal cord in American whites and American Negroes. Anat Rec, 63: 417-424.

16. Patil DS, Jadav Hrishikesh R, Binodkumar, Mehta CD, Patel Vipul D (2012) Anatomical study of sacral hiatus for caudal epidural block. Nat J Med Res, 2: 272-275.
17. Phongkitkarum S, Jaovisidha S, Dhanachai M (2004) Determination of the thecal sac ending using magnetic resonance imaging: clinical applications in craniospinal irradiation. J Med Assoc Thai, 87: 1368-1373.

18. Pinto FC, Fontes RB, Leonhardt Mde C, Amodio DT, Porro FF, Machado J (2002) Anatomic study of the filum terminale and its correlations with the tethered cord syndrome. Neurosurgery 51: 725-730.

19. Rahmani M, Shabani Samghabadi MA, Vaziri Bozorg SM (2009) Magnetic resonance imaging based determination of conus medullaris position in adults. Res J Biol Scien, 4: 157-159.

20. Reiman AF, Anson BJ (1944) Vertebral level of termination of the spinal cord with report of a case of a sacral cord. Anat Rec, 88: 127-138.

21. Saifuddin A, Burnett SJD, White J (1998) The variation of position of the conus medullaris in an adult population. A magnetic resonance imaging study. Spine, 23: 1452-1456.

22. Scharf CB, Paulino AC, Goldberg KN (1998) Determination of the inferior border of the thecal sac using magnetic resonance imaging: implications on radiation therapy treatment planning. Int J Radiat Oncol Biol Phys, 41: 621-624.

23. Sekiguchi M, Yabuki S, Saton K, Kikuchi S (2004) An anatomical study of the sacral hiatus: a basis for successful caudal epidural block. Clin J Pain, 20: 51-54.

24. Senoglu N, Senoglu M, Oksuz H, Gumusalan Y, Yukse KZ, Zencirci B, Ezberci M, Kizillkanat E (2005) Landmarks of the Sacral hiatus for caudal epidural block: an anatomical study. Br J Anaesth, 95: 692-695.

25. Soleiman J, Demaerel P, Rocher S, Maes F, Marchal G (2005) Magnetic resonance imaging study of the level of termination of the conus medullaris and the thecal sac: influence of age and gender. Spine, 30: 1875-1880.

26. Standring S (2008) Gray's anatomy. The anatomical basis of clinical practice. 40th Ed. Churchill Livingstone Elsevier, London, pp. 749-761.

27. Waldman SD (2004) Caudal epidural block: prone position. In: Atlas of interventional pain management. $2^{\text {nd }} \mathrm{Ed}$. Saunders, Philadelphia, pp. 380-392.

28. Wilson DA, Prince JR (1989) MR imaging determination of the location of the normal conus medullaris throughout childhood. Am J Neuroradiol, 10: 259-262. 